\title{
Lung cancer with spontaneous regression of primary and metastatic sites: A case report
}

\author{
RYOKO OGAWA ${ }^{1}$, HIROKO WATANABE ${ }^{1}$, KAI YAZAKI ${ }^{1}$, KAZUTAKA FUJITA ${ }^{1}$, YOSHIYA TSUNODA ${ }^{1}$, \\ KENSUKE NAKAZAWA ${ }^{1}$, SHINSUKE HOMMA ${ }^{1}$, KATSUNORI KAGOHASHI ${ }^{2}$, \\ HIROAKI SATOH $^{2}$ and NOBUYUKI HIZAWA ${ }^{1}$ \\ ${ }^{1}$ Division of Respiratory Medicine, Faculty of Medicine; ${ }^{2}$ Division of Respiratory Medicine, \\ Mito Medical Center, University of Tsukuba, Mito, Ibaraki 310-0015, Japan
}

Received August 13, 2014; Accepted April 30, 2015

DOI: $10.3892 / \mathrm{ol} .2015 .3243$

\begin{abstract}
Partial or complete spontaneous cancer regression is a rare phenomenon, particularly in patients with lung cancer. This is the case report of a patient with lung cancer who exhibited spontaneous regression of the primary as well as metastatic lesions, without receiving any treatment. Spontaneous regression commenced within a week of obtaining pathological specimens by transbronchial and percutaneous biopsies from the primary lesion and metastatic lymph nodes of the left side of the neck. The reason for this phenomenon is unknown; however, we hypothesized that there may be an immunological association between the stimulus of the biopsies and the spontaneous regression. This patient should be closely followed up to monitor the clinical course of this unusual case.
\end{abstract}

\section{Introduction}

Spontaneous regression has been reported in a variety of malignant diseases, including lung cancer, whereas the types of cancer most commony exhibiting this rare phenomenon include malignant melanoma, neuroblastoma and renal cell carcinoma (1-3). The standard definition of spontaneous regression is 'the partial or complete disappearance of a malignant tumor in the absence of treatment or in the presence of therapy considered inadequate to exert a significant influence on the disease' $(1,2)$. The distinct pathogenetic mechanism underlying spontaneous regression in malignant tumors remains unclear, as there is no consensus due to its rare occurrence. This is the case report of a patient with lung

Correspondence to: Professor Hiroaki Satoh, Division of Respiratory Medicine, Mito Medical Center, University of Tsukuba, Miya-machi 3-2-7, Mito, Ibaraki 310-0015, Japan

E-mail: hirosato@md.tsukuba.ac.jp

Key words: lung cancer, spontaneous regression, primary tumor, metastasis cancer who exhibited spontaneous regression of the primary and metastatic lesions without having received treatment. The patient provided written informed consent.

\section{Case report}

A 65-year-old man was referred to Mito Medical Center, University of Tsukuba (Mito, Japan) with a mass in the left side of the neck. The tumor had enlarged rapidly over the previous 2 weeks and was accompanied by severe pain in the left arm. Within a week from the first presentation, paralysis developed gradually below the level of $\mathrm{T} 9$ and the patient was admitted to our hospital. The patient was a heavy smoker, with no family or past history of disease. At the first presentation, the physical examination revealed a sizeable, hard, fixed tumor on the left side of the neck. The laboratory examination revealed a white blood cell count of 9,800/ $\mu 1$, a C-reactive protein level of $8.59 \mathrm{mg} / \mathrm{dl}$ and a cytokeratin-19 fragments level of $5.3 \mathrm{ng} / \mathrm{ml}$. A computed tomography (CT) scan of the neck revealed enlarged, bulging left cervical lymph nodes (Fig. 1A). A chest radiograph and CT scan revealed a solid peripheral tumor in the right lung with enlarged lymph nodes on both sides of the mediastinum (Figs. 2A and 3A). Magnetic resonance imaging (MRI) of the thoracic vertebrae revealed metastasis to the vertebral body and dura mater in T9. The findings of the abdominal CT and brain MRI scans were normal.

Percutaneous aspiration cytology of the tumor on the left side of the neck and transbronchial biopsy of the upper lobe of the right lung were performed and the pathological examination established the diagnosis of poorly differentiated non-small-cell lung cancer, with metastases to the cervical lymph nodes. A week after these biopsies, spontaneous regression of the neck tumor was identified on a CT scan of the neck (Fig. 1B) and regression of the primary tumor of the lung and mediastinal lymph nodes was observed on chest radiography. One month after the first presentation, spontaneous regression of the primary tumor and mediastinal lymph nodes was confirmed by chest radiograph and CT scan (Figs. 2B and 3B). Thereafter, the patient underwent irradiation of the vertebra and dura mater at the T9 metastatic site. Despite receiving no additional therapy for the primary tumor or the tumor of the neck, the size of these lesions continued to decrease, almost 

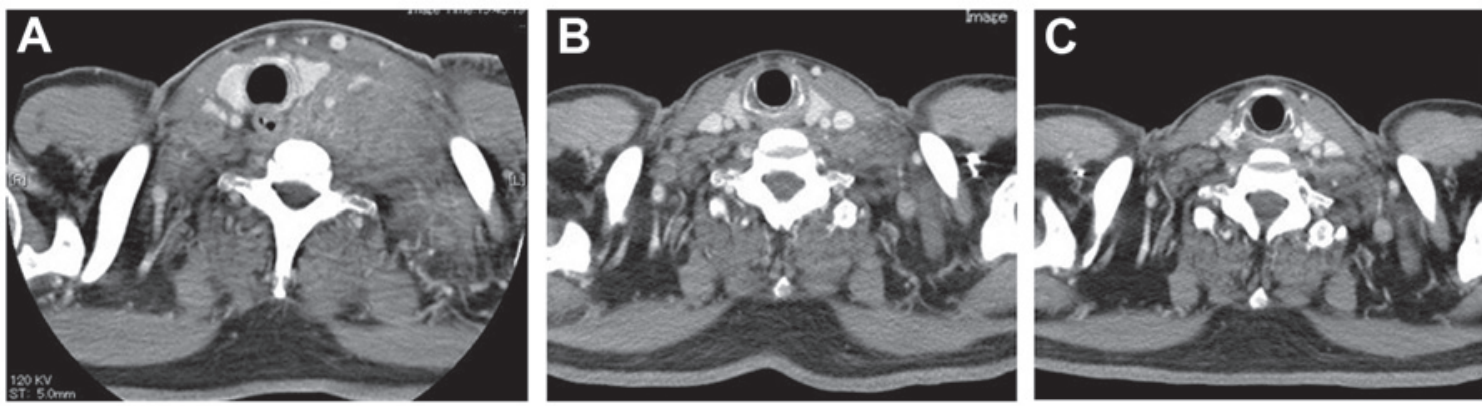

Figure 1. Computed tomography (CT) scans of the neck. (A) The CT scan at first presentation revealed a sizeable bulging mass on the left side of the neck. (B) The follow-up CT scan revealed spontaneous regression of the left cervical lymph nodes 1 month after the first presentation. (C) Almost complete disappearance of the cervical lymph node metastasis 2 months after the first presentation.
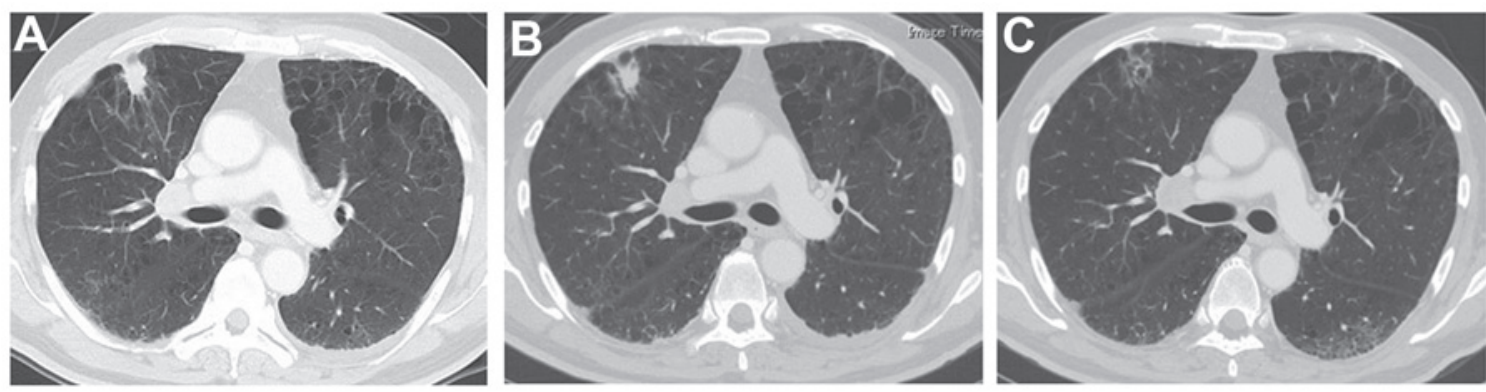

Figure 2. Chest computed tomography (CT) scans. (A) The chest CT scan at first presentation revealed a tumor in the right lung. (B) The follow-up chest radiograph revealed spontaneous regression of the tumor 1 month after the first presentation. (C) Almost complete disappearance of the tumor 2 months after the first presentation.
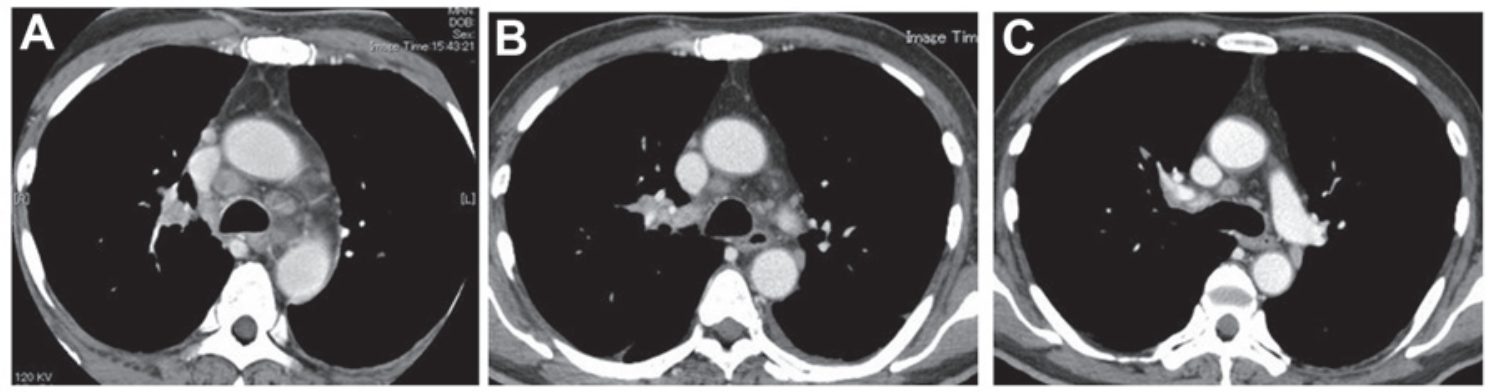

Figure 3. Chest computed tomography (CT) scans. (A) The chest CT scan at first presentation revealed bilateral mediastinal lymph node enlargement. (B) The follow-up chest CT scan revealed spontaneous regression of the mediastinal lymph nodes 1 month after the first presentation. (C) Almost complete disappearance of the mediastinal lymph node metastasis 2 months after the first presentation.

disappearing completely 2 months after the first presentation (Figs. 1C, 2C and 3C).

\section{Discussion}

Lung cancer metastasis to vascular- and lymphatic-associated organs is frequent and the most common metastatic sites are the lung, bone and brain (4-6). Lymph node involvement is primarily regional in cancers of the internal organs, including lung cancer $(4,7)$. Therefore, common sites of lymph node metastasis in lung cancer are the hilar, mediastinal and supraclavicular nodes. Metastasis to the cervical lymph nodes and thoracic vertebra are occasionally observed at advanced stages, suggesting that cancer cells may reach several sites throughout the body via the bloodstream and lymphatic system.
Spontaneous regression of malignant disease, according to a generally accepted definition, is a complete or partial, temporary or permanent disappearance of all or at least certain relevant parameters of a soundly diagnosed malignant disease, without any medical treatment or with treatment that is considered inadequate to produce the resulting regression $(1,2)$. The present case may be classified as a partial spontaneous regression according to this definition. Everson and Cole (2) reported only 176 cases of spontaneous regression between 1900 and 1964 , with an estimated incidence of 1/60,000-100,000 cancer patients. Challis and Stam (3) updated that review. According to their review, 741 cases of spontaneous regression of malignant diseases were reported in the literature between 1900 and 1987 (3). Although a variety of malignant diseases were reported, renal cell carcinoma, neuroblastoma, malignant 
melanoma, choriocarcinoma, bladder cancer, retinoblastoma, lymphoma, leukemia and breast cancer accounted for two-thirds of all the cases (3). Despite its incidence in a variety of malignant diseases, spontaneous regression in lung cancer is considered to be a particularly rare event $(1,2,8,9)$. Everson and Cole (2) reported only two cases, whereas Lowy and Erickson had only found four previous cases in the medical literature when they reported an additional case of spontaneous regression in a patient with metastatic small-cell lung cancer in 1986 (10).

In the present case, the histological diagnosis of lung cancer was established by specimens obtained during the transbronchial procedure; the neck lesion was also histologically confirmed to be metastatic from lung cancer by specimens obtained during the skin biopsy. Although histological confirmation of metastatic lung adenocarcinoma was obtained, the metastatic neck lesion disappeared shortly following the skin biopsy. Certain previous studies have reported spontaneous regression of metastatic lesions following removal of the primary lesion (11-15), whereas other studies have reported spontaneous regression associated with infection $(1,16)$. In the present case, however, spontaneous regression of the metastatic lesion was observed without any treatment of the primary or metastatic lesions; in addition, there was no infection of the primary or metastatic sites. It was recently reported that immunological mechanisms, including certain cytokines, such as interferon and tumor necrosis factor, may stimulate cytotoxic function against cancer cells, leading to spontaneous regression (17-19). Unfortunately, blood samples were not obtained for immunological evaluation, as there was no expectation of tumor regression at the time. Therefore, data elucidating the precise immunological association are lacking and the exact mechanism of spontaneous regression of the metastatic lesion remains unknown.

We previously reported the case of a patient with lung adenocarcinoma exhibiting spontaneous regression of a scalp metastasis (15). Notably, in that previous case, spontaneous regression of the scalp metastatic lesion developed after 1 month following biopsy of the scalp mass and the regression of the primary lung lesion also developed 1 month following a transbronchial biopsy (15). In the present case, spontaneous regression occurred not only in the cervical lymph node metastatic lesion, but also in the primary lung lesion 1 month after obtaining the pathological specimens. It is noteworthy that, in these two patients, spontaneous regression developed shortly after a direct invasive approach to the tumor lesions, which is highly suggestive of an association between the onset of the regression and a change in the intratumoral immunological mechanism between the host and the tumor. A likely explanation is that there may be a stimulus associated with the direct invasive approach to the lesions, which initiates the spontaneous regression. However, the precise mechanism remains unclear and future studies are required to elucidate this mechanism. The patient should be closely followed up to monitor the clinical course of this unusual case. A later report may reveal the causes and broaden the knowledge regarding this uncommon phenomenon.

\section{References}

1. Cole WH: Effects to explain spontaneous regression of cancer. J Surg Oncol 17: 201-209, 1981.

2. Everson TC and Cole WH: Spontaneous regression of cancer. Philadelphia WB Sounders, 1966.

3. Challis GB and Stam HJ: The spontaneous regression of cancer. A review of cases from 1900-1987. Acta Oncol 29: 545-550, 1990.

4. Gueron G, De Siervi A and Vazquez E: Key questions in metastasis: new insights in molecular pathways and therapeutic implications. Curr Pharm Biotechnol 12: 1867-1880, 2011

5. Oikawa A, Takahashi H, Ishikawa H, Kurishima K, Kagohashi K and Satoh H: Application of conditional probability analysis to distant metastases from lung cancer. Oncol Lett 3: 629-634, 2012.

6. Nakazawa K, Kurishima K, Tamura T, et al: Specific organ metastases and survival in small cell lung cancer. Oncol Lett 4: 617-620, 2012.

7. Sampath L, Kwon S, Hall MA, Price RE and Sevick-Muraca EM Detection of cancer metastases with a dual-labeled near-infrared/positron emission tomography imaging agent. Transl Oncol 3: 307-317, 2010.

8. Kappauf H, Gallmeier WM and Wunch PH: Complete spontaneous remission in a patient with metastatic non-small-cell lung cancer. Case report, review of literature and discussion of possible biological pathways involved. Ann Oncol 8: 1031-1039, 1997.

9. Cafferata MA, Chiaramondia M, Monetti F and Ardizzoni A: Complete spontaneous remission of non-small-cell lung cancer: a case report. Lung Cancer 45: 263-266, 2004.

10. Lowy AD Jr and Erickson ER: Spontaneous 19-year regression of oat cell carcinoma with scalene metastasis. Cancer 58: 978-980, 1986.

11. Deweerd JH, Hawthorne NJ and Adson MA: Regression of renal cell hepatic metastasis following removal of primary lesions. J Urol 117: 790-792, 1977.

12. Marces SG, Choyke PL, Reiter R, et al: Regression of metastatic renal cell carcinoma after cytoreductive nephrectomy. J Urol 150: 463-466, 1993

13. Gohji K, Kizaki T and Fujii A: Spontaneous regression of pulmonary metastasis from nonfunctioning adrenocortical carcinoma after removal of the primary lesion: a case report. J Urol 154: 1854-1855, 1995.

14. Hammad AM, Paris GR, van Heuven WA, Thompson IM and Fitzsimmons TD: Spontaneous regression of choroidal metastasis from renal cell carcinoma. Am J Ophthalmol 135: 911-913, 2003.

15. Miyazaki K, Masuko H, Satoh H and Ohtsuka M: Lung cancer with spontaneous regression of scalp metastasis. Respir Med Extra 3: 83-85, 2007.

16. Wadsworth SJ, Davies CW, Gray W and Gleeson FV: Spontaneous regression of pulmonary metastases demonstrated by $\mathrm{CT}$. Br J Radiol 72: 304-307, 1999.

17. Stoll BA: Spontaneous regression of cancer: new insights. Biotherapy 4: 23-30, 1992.

18. Moroda T, Iiai T and Suzuki S: Autologous killing by a population of intermediate T-cell receptor cells and its NK1.1+ and NK1.1 subsets, using Fas ligand/Fas molecules. Immunology 91: 219-226, 1997.

19. Kawamura T, Seki S and Takeda K: Protective effect of NK1.1 cells as well as NK cells against intraperitoneal tumors in mice. Cell Immunol 193: 219-225, 1999. 